\title{
Consumo de té y cáncer. Revisión sistemática metaanálisis
}

\author{
Celia Puig Moliner \\ al339193@uji.es \\ Adrián Fonta de la Fuente \\ al339194@uji.es \\ Mar Cerda Peraire \\ al339179@uji.es \\ Ana Folch Ayora \\ afolch@uji.es
}

\section{Resumen}

Introducción: El té es una de las bebidas más antiguas y populares que se consumen en todo el mundo, posee propiedades antiinflamatorias y antioxidantes. Su consumo se ha asociado con una reducción o aumento de la incidencia de determinados tipos de cánceres.

Objetivos: Conocer la relación existente entre el consumo de té y el cáncer en población con antecedentes o diagnóstico de cáncer.

Metodología: Revisión sistemática de la literatura en las bases de datos Pubmed (Medline), ProQuest Nursing \& Allied Health database, CINAHL, Lilacs y Biblioteca Cochrane Plus, utilizando la estrategia de búsqueda Tea AND Neoplasms con los filtros ensayo clínico, últimos 10 años y humanos. Evaluación calidad metodológica mediante la herramienta Critical Appraisal Skills Programme Español, con puntuación CASPe $\geq 6$.

Resultados: Un total de $\mathrm{N}=14$ estudios cumplieron los criterios de inclusión. Los cánceres más estudiados fueron el de próstata $(n=7)$, mama $(n=2)$, ginecológicos (endometrio y ovario) $(n=2)$ y otros tumores (hígado, colón, oral) $(n=3)$. El consumo de té presentó una correlación positiva con el cáncer de próstata $(n=5)$, de mama $(n=1)$ y de hígado $(n=1)(p<0,05)$.

Conclusión: El té puede ser una bebida con propiedades anticancerígenas. Pero dada la heterogeneidad de los tumores estudiados y la diversidad de variables estudiadas, no se han podido establecer conclusiones generales, siendo necesario un mayor número de investigaciones.

Palabras clave: té, neoplasia, prevención de cáncer, polifenoles. 


\begin{abstract}
Introduction: Tea is one of the oldest and most popular beverages consumed worldwide, it has anti-inflammatory and antioxidant properties. Its consumption has been associated with a reduction or increase in the incidence of certain types of cancer.

Objectives: The main aim of this review is to know the relationship between tea and cancer in the population with a history or diagnosis of cancer.

Methodology: Systematic review of the literature in the databases: Pubmed (Medline), ProQuest Nursing \& Allied Health database, CINAHL, Lilacs, Cochrane Library using search strategy "Tea" AND "Neoplasms" with clinical trial, last 10 years and human filters. Methodological quality evaluation using the Critical Appraisal Skills Program Spanish tool, with CASPe score $\geq 6$.

Results: A total of $\mathrm{N}=14$ studies met the inclusion criteria. The most studied tumors were prostate $(n=7)$, breast $(n=2)$, gynaecological (endometrium and ovary) $(n=2)$ and other tumors (liver, colon, oral) $(n=3)$. Tea consumption was associated with prostate cancer $(n=5)$, breast cancer $(n=1)$ and liver cancer $(n=1),(p<0.05)$.

Conclusion: Tea may be a beverage with anti-cancer properties. However, given the heterogeneity of the tumors studied and the diversity of variables studied, it has not been able to establish general conclusions, and more research is needed.
\end{abstract}

Keywords: tea, neoplasms, cancer prevention, polyphenols.

\title{
Introducción
}

El té es una de las bebidas de mayor popularidad y tradición en el mundo. Del total de té producido y consumido en el mundo, el $78 \%$ es negro y el $20 \%$ es verde (Khan y Mukhtar 2018). Independientemente de la variedad del té, todos ellos emanan de las hojas de la planta Camellia Sinensis. La cantidad de oxidación y otros aspectos del método poscosecha determinan el tipo de té que se va a producir (Peterson et al. 2004). En el caso del té verde, las hojas frescas se procesan rápidamente para inactivar las enzimas, lo que evita la oxidación y produce un producto seco y estable. En cambio, el té negro se elabora triturando y secando hojas de té frescas hasta que su contenido de humedad se reduce para efectuar la fermentación y oxidación completa antes del procesamiento final (Mukhtar y Ahmad 2000). Así pues, pese a existir diversas variedades de té, todas contienen en diferentes proporciones trazas de proteínas, carbohidratos, aminoácidos, alcaloides (cafeína, teofilina y teobromina), vitaminas, minerales (aluminio), oligoelementos, así como una amplia gama de compuestos químicos como los polifenoles y compuestos orgánicos volátiles que le aportan el olor y el aroma (Cabrera, Giménez y López 2003), responsables de los principales efectos beneficiosos que tiene el té para la salud. Las dietas deficientes en polifenoles se relacionan con un mayor riesgo de padecer cáncer. Su base científica radica en las propiedades de los polifenoles en la captación de radicales libres y en la inhibición de células que superan su capacidad de división, desencadenando la apoptosis (Peterson et al. 2004).

Por lo tanto, esta enfermedad es de gran importancia por su elevada incidencia y mortalidad en el mundo. La prevención de la misma y la supervivencia de los pacientes que la padecen se basan en la reducción de la exposición a factores de 
riesgo. Siendo los hábitos dietéticos los causantes del 30 al $35 \%$ de los cánceres, es importante conocer el papel que ejerce el consumo de té en la salud de la población y la posible influencia sobre el cáncer. Por tanto, el objeto general de esta revisión sistemática es conocer la relación existente entre el té y el cáncer en la población con antecedentes o diagnóstico de cáncer.

\section{Metodología}

\section{Diseño}

Revisión sistemática de la literatura científica sobre la relación existente entre el té y el cáncer en la población, disponible en las bases de datos Pubmed (Medline), Literatura Latinoamericana y del Caribe en Ciencias de la Salud (LILACS), Cumulative Index to Nursing \& Allied Health Literature (CINAHL), ProQuest Nursing \& Allied Health Source y Cochrane Library.

\section{Estrategia de búsqueda}

En primer lugar, se formuló la pregunta de investigación: ¿Qué efecto tiene el consumo de té en la población con antecedentes de cáncer, con lesiones precursoras y con diagnóstico de cáncer? Después, se seleccionaron las palabras clave que contenían nuestra pregunta de investigación, haciendo uso de los Descriptores en Ciencias de la Salud (DeCS) y los descriptores del Medical Subject Headings (MeSH). La estrategia de búsqueda quedó constituida por los descriptores Neoplasms y Tea utilizando el operador booleano $A N D$ entre ambos. Para la delimitación de esta búsqueda se aplicaron varios filtros, como criterios de temporalidad (10 últimos años) $y$, en referencia a la tipología de estudio, se seleccionaron únicamente ensayos clínicos. Así pues, para la base de datos Pubmed (Medline) se incluyó en la estrategia de búsqueda el acceso a texto completo. $Y$ para Proquest se incluyó el filtro de artículos principales.

\section{Criterios de selección}

Criterios de inclusión: ensayos clínicos, humanos, criterios de temporalidad de publicación en los últimos 10 años, acceso a texto completo, calidad metodológica $\geq 6$.

Criterios de exclusión: artículos ya incluidos/repetidos, artículos cuyo objetivo no presentaba relación entre el consumo de té con el cáncer, población infantil o adolescente, individuo menor de 18 años, artículos que abordaban el efecto de otros compuestos derivados del té.

\section{Proceso de selección}

Una vez obtenidos los artículos resultantes mediante la estrategia de búsqueda, se procede a su lectura teniendo en cuenta los criterios establecidos, se excluyen o aceptan en función del objeto de estudio de esta revisión, para su posterior análisis. 


\section{Variables del estudio}

Características sociodemográficas: sexo, edad.

Aspectos relacionados con el cáncer: tipo de tumor, estado del paciente (alto riesgo, lesiones premalignas, cáncer localizado), estadio tumoral.

Aspectos relacionados con el té:

-Variedad de tipo de té.

-Medida de la ingesta de té.

-Medición y complicaciones de la ingesta.

Relación del té con el cáncer: principales resultados y significación estadística.

\section{Valoración de la calidad metodológica}

Los artículos seleccionados tras la lectura del texto completo fueron sometidos a la valoración de su calidad metodológica mediante la herramienta Critical Appraisal Skills Programme Español (CASPe) para la valoración de ensayos clínicos aleatorizados. Dicha escala consta de 11 ítems, siendo los 3 primeros de exclusión y los 7 siguientes complementarios. Así pues, se atribuyen dos criterios de exclusión: el primero, el no cumplimiento de los 3 primeros ítems, y el segundo, la no obtención de una puntuación $\geq 6$ sobre 11 .

\section{Resultados}

Resultados de la búsqueda y del proceso

Usando la estrategia de búsqueda planteada se obtuvieron un total de $n=2172$ resultados, que fueron reducidos a $n=231$ tras aplicar los filtros de temporalidad y de tipología de estudio. Mediante la realización de un cribado manual usando los criterios de selección se incluyeron un total de 14 artículos. Dichos resultados fueron representados mediante el diagrama PRISMA. Véase la figura 1.

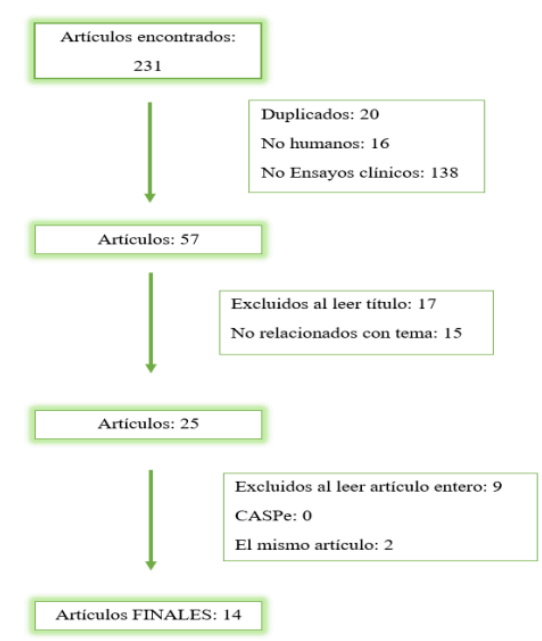

Figura 1. Diagrama de flujo del proceso PRISMA. 


\section{Características sociodemográficas}

El $57 \%(n=8)$ de los estudios fueron hombres, el $29 \%(n=4)$ mujeres y ambos sexos en el $14 \%(n=2)$. Con respecto a la edad, la población a estudio estuvo reflejada en $\mathrm{n}=12$ estudios, cuyos resultados muestran que la edad se situó en torno a los 28,4 y 74 años, situándose la media en 59,4 años. Véanse los datos en la tabla 1.

Tabla 1

Características sociodemográficas

\begin{tabular}{|l|l|l|l|}
\hline Artículos & Sexo & Rango edades (años) & Edad media (años) \\
\hline Samavat et al. 2017 & Mujeres & $50-70$ & 59.8 \\
\hline Van Die et al. 2017 & Hombres & - & 72 \\
\hline Xue et al. 2015 & Ambos & $20-52$ & - \\
\hline Kumar et al. 2015 & Hombres & $30-80$ & 63.1 \\
\hline Henning et al. 2015 & Hombres & $40-70$ & 62.1 \\
\hline Crew et al. 2015 & Mujeres & $21-65$ & 55 \\
\hline Thomas et al. 2014 & Hombres & $53-89$ & 74 \\
\hline Garcia et al. 2014 & Mujeres & $18-58$ & 28.4 \\
\hline Trudel et al. 2013 & Mujeres & - & 59.6 \\
\hline Henning et al. 2013 & Hombres & $54-69$ & 61 \\
\hline Nguyen et al. 2012 & Hombres & - & 62.3 \\
\hline Wang et al. 2010 & Hombres & - & - \\
\hline Tsao et al. 2009 & Ambos & $33-76$ & 57 \\
\hline McLarty et al. 2009 & Hombres & $41-68$ & 58.5 \\
\hline
\end{tabular}

\section{Aspectos relacionados con el cáncer}

Los tumores más estudiados fueron el de próstata $46 \%(n=7)$, mama $13 \%(n=2)$, ginecológicos (endometrio y ovario) $13 \%(n=2)$ y el de hígado $13 \%(n=2)$. En el $65 \%$ $(n=9)$ de los estudios se hace referencia a pacientes diagnosticados de cáncer. El $21 \%(n=3)$ de los estudios hacen referencia a pacientes con lesiones premalignas y en un $7 \%(n=1)$ a pacientes con alta probabilidad de desarrollar un tumor pero sin lesiones. Así pues, de los $n=9$ que presentaban un cáncer localizado, el estadio tumoral no fue identificado en el $65 \%(n=6)$ de los estudios. Dentro de los que sí fue identificado, en el $22 \%(n=2)$ incluyeron participantes con un estadio tumoral I-III y un $11 \%(n=1)$ con estadio III-IV (véase la tabla 2$)$.

Tabla 2

Aspectos relacionados con el cáncer

\begin{tabular}{|l|l|l|l|}
\hline Artículos & Tipo de cáncer & Estado del paciente & Estadio tumoral \\
\hline Samavat et al. 2017 & Mama e hígado & Alta probabilidad & \\
\hline Van Die et al. 2017 & Próstata & Diagnosticado & - \\
\hline Xue et al. 2015 & Hígado & - & \\
\hline Kumar et al. 2015 & Próstata & Lesiones premalignas & \\
\hline Henning et al. 2015 & Próstata & Diagnosticado & - \\
\hline Crew et al. 2015 & Mama & Diagnosticado & I-III \\
\hline Thomas et al. 2014 & Próstata & Diagnosticado & - \\
\hline Garcia, Francisco et al. 2014 & Cervical & Lesiones premalignas & \\
\hline Trudel et al. 2013 & Ovario & Diagnosticado & III-IV \\
\hline Henning et al. 2013 & Colón & Diagnosticado & - \\
\hline
\end{tabular}




\begin{tabular}{|l|l|l|l|}
\hline Artículos & Tipo de cáncer & Estado del paciente & Estadio tumoral \\
\hline Wang et al. 2010 & Próstata & Diagnosticado & - \\
\hline Tsao et al. 2009 & Oral & Lesiones premalignas & \\
\hline McLarty et al. 2009 & Próstata & Diagnosticado & I-III \\
\hline
\end{tabular}

\section{Aspectos relacionados con el té}

El $71 \%(n=10)$ de los estudios hicieron referencia a la ingesta diaria de cápsulas con los componentes principales del té. La media de la ingesta en los estudios fue de $620 \mathrm{mg} / \mathrm{día}$. En el $71 \%(\mathrm{n}=10)$ de los estudios valoraron el consumo de té en base al número de cápsulas al día y en el $29 \%(n=4)$ restante hablaban del consumo de tazas de té diarias, con una media en el $100 \%(n=4)$ de 6 tazas diarias. En todos los estudios se obtuvieron muestras sanguíneas para el análisis de las variables y se realizaron otros procedimientos como la obtención de muestras de orina $(n=11)$, biopsias $(n=6)$, análisis antropométricos $(n=1)$, mamografía $(n=1)$ y examen de recto y vagina $(n=1)$. En $n=4$ estudios se habla de reacciones a la teína como: la inquietud, el insomnio y el nerviosismo. En $n=5$ estudios se hace referencia a alteraciones de la función hepática y en $n=5$ de alteraciones gastrointestinales como acidez, náuseas, vómitos y estreñimiento. Finalmente, en $n=4$ no hicieron referencia a ningún tipo de anomalía (véase la tabla 3).

Tabla 3

Aspectos relacionados con el té

\begin{tabular}{|c|c|c|c|}
\hline Artículos & Presentación & Cantidad & Complicaciones \\
\hline Samavat et al. 2017 & Cápsulas & $800 \mathrm{mg} / \mathrm{día}$ & $\begin{array}{l}\text { Anomalías función } \\
\text { hepática }\end{array}$ \\
\hline Van Die et al. 2017 & $\begin{array}{l}\text { Cápsulas } \quad(50 \% \\
\text { té) }\end{array}$ & 400 mg/día & $\begin{array}{l}\text { Reacciones teína } \\
\text { (inquietud); } \\
\text { gastrointestinales (acidez) }\end{array}$ \\
\hline Xue et al. 2015 & Cápsulas & $\begin{array}{l}500 \mathrm{mg} / \mathrm{día} \\
1000 \mathrm{mg} / \mathrm{di} a\end{array}$ & Nada \\
\hline Kumar et al. 2015 & Cápsulas & 400 mg/día & $\begin{array}{ll}\text { Anomalías } & \text { función } \\
\text { hepática } & \end{array}$ \\
\hline Henning et al. 2015 & Tazas & 6 tazas/día & $\begin{array}{l}\text { Reacciones teína } \\
\text { gastrointestinales }\end{array}$ \\
\hline Crew et al. 2015 & Cápsulas & $\begin{array}{l}400 \mathrm{mg} / \mathrm{día} \\
600 \mathrm{mg} / \mathrm{día} \\
800 \mathrm{mg} / \mathrm{día}\end{array}$ & $\begin{array}{l}\text { Anomalías función } \\
\text { hepática; reacciones teína } \\
\text { (insomnio) }\end{array}$ \\
\hline Thomas et al. 2014 & Cápsulas & 100 mg/día & Gastrointestinales \\
\hline Garcia et al. 2014 & Cápsulas & 800 mg/día & $\begin{array}{lr}\text { Anomalías } & \text { función } \\
\text { hepática; gastrointestinales } \\
\text { (náuseas) }\end{array}$ \\
\hline Trudel et al. 2013 & Tazas & $500 \mathrm{ml} / \mathrm{día}$ & Gastrointestinales \\
\hline Henning et al. 2013 & Tazas & 6 tazas/día & Nada \\
\hline Nguyen et al. 2012 & Cápsulas & 800 mg/día & $\begin{array}{l}\text { Anomalías } \\
\text { hepática }\end{array}$ \\
\hline Wang et al. 2010 & Tazas & 6 tazas/día & Nada \\
\hline Tsao et al. 2009 & Cápsulas & $\begin{array}{l}500 \mathrm{mg} / \text { día } \\
750 \mathrm{mg} / \mathrm{día} \\
1000 \mathrm{mg} / \mathrm{día}\end{array}$ & $\begin{array}{l}\text { Reacciones } \\
\text { (insomnio) }\end{array}$ \\
\hline McLarty et al. 2009 & Cápsulas & 800/ día & Nada \\
\hline
\end{tabular}




\section{Relación del té con el cáncer}

El grado de significación de los resultados de interés en este estudio varió entre 0,80 y $<0,0008$. En un $57 \%(n=8)$ de los estudios hubo un grado de significación estadística por debajo de 0,05. Asimismo, en el $14 \%(n=2)$ de los estudios se observa un grado de significación estadística menor de 0,01 (véase la tabla 4).

Tabla 4

Te y cáncer

\begin{tabular}{|c|c|c|c|c|}
\hline Artículos & Resultados & $\begin{array}{l}\text { Relación } \\
\text { cáncer }\end{array}$ & $\begin{array}{l}\text { Cáncer } \\
\text { estudiado }\end{array}$ & $P$ value \\
\hline $\begin{array}{l}\text { Samavat } \\
\text { et al. } 2017\end{array}$ & $\begin{array}{l}\text { Suplementos con polifenoles de té } \\
\text { redujeron significativamente la densidad } \\
\text { mamográfica en las mujeres más jóvenes. } \\
\text { Interacción entre la edad y la } \\
\text { suplementación con té. }\end{array}$ & $\begin{array}{l}\text { 1.SÍ } \\
\text { 2.NO }\end{array}$ & $\begin{array}{l}\text { 1. mama } \\
\text { 2. hígado }\end{array}$ & $P=0,05$ \\
\hline $\begin{array}{l}\text { Van Die et } \\
\text { al. } 2017\end{array}$ & $\begin{array}{l}\text { Tiempos de duplicación de } \mathrm{PSA}_{1} \text { no } \\
\text { significativos. Cambio en la pendiente del } \\
\text { logaritmo } \mathrm{PSA}_{1} \text { no significativo. }\end{array}$ & NO & próstata & $P=0,21$ \\
\hline $\begin{array}{l}\text { Xue et al. } \\
2015\end{array}$ & $\begin{array}{l}\text { Los biomarcadores urinarios, la proporción } \\
\text { libre de fumonisina B1 y Sa/So } \\
\text { disminuyeron significativamente en los } \\
\text { grupos de tratamiento con té y el efecto fue } \\
\text { mayor con el tiempo. }\end{array}$ & sí & hígado & $p<0,016$ \\
\hline $\begin{array}{l}\text { Kumar et } \\
\text { al. } 2015\end{array}$ & $\begin{array}{l}\text { El criterio de valoración principal de este } \\
\text { estudio no se cumplió ya que no se } \\
\text { observaron diferencias significativas en las } \\
\text { tasas de } \mathrm{PSA}_{1} \text { entre los brazos de estudio. } \\
\text { Análisis secundario: eliminando hombres } \\
\text { con ASAP } \mathrm{P}_{2} \text { se observó una disminución en } \\
\text { la } \mathrm{PSA}_{1} \text {. }\end{array}$ & sí & próstata & $P<0,024$ \\
\hline $\begin{array}{l}\text { Henning et } \\
\text { al. } 2015\end{array}$ & $\begin{array}{l}\text { El consumo de té negro no se asoció con } \\
\text { ningún efecto significativo. En el consumo } \\
\text { de té verde hubo niveles detectables en } \\
\text { tejido prostático en el } 94 \% \text { de los } \\
\text { participantes. }\end{array}$ & sí & próstata & $P<0,05$ \\
\hline $\begin{array}{l}\text { Crew et al. } \\
2015\end{array}$ & $\begin{array}{l}\text { Ningún cambio significativo en la densidad } \\
\text { mamográfica. Mecanismos de acción en la } \\
\text { angiogénesis y el metabolismo de los } \\
\text { lípidos. }\end{array}$ & sí & mama & $P<0,001$ \\
\hline $\begin{array}{l}\text { Thomas et } \\
\text { al. } 2014\end{array}$ & $\begin{array}{l}\text { El } \mathrm{PSA}_{1} \text { promedio aumentó desde el inicio } \\
\text { hasta el final de la intervención. El aumento } \\
\text { de } \mathrm{PSA}_{1} \text { de forma más lenta se dio en el } \\
\text { grupo intervenido con cápsulas. }\end{array}$ & sí & próstata & $P=0,0008$ \\
\hline $\begin{array}{l}\text { Garcia et } \\
\text { al. } 2014\end{array}$ & $\begin{array}{l}\text { El estado positivo del virus del papiloma } \\
\text { humano al final del estudio era similar entre } \\
\text { los dos grupos. Los resultados histológicos } \\
\text { fueron similares en ambos grupos. }\end{array}$ & NO & cervical & $P=0,80$ \\
\hline $\begin{array}{l}\text { Trudel et } \\
\text { al. } 2013\end{array}$ & $\begin{array}{l}\text { Detener el ensayo clínico después de la } \\
\text { primera etapa ya que no se tenían pruebas } \\
\text { para rechazar la hipótesis nula. Toxicidades } \\
\text { informadas. }\end{array}$ & NO & ovario & - \\
\hline $\begin{array}{l}\text { Henning et } \\
\text { al. } 2013\end{array}$ & $\begin{array}{l}\text { Aumento de marcadores urinarios y séricos. } \\
\text { Aumento significativo en la inhibición de la } \\
\text { proliferación de células de cáncer de colón. }\end{array}$ & sí & Colón & $P<0,13$ \\
\hline
\end{tabular}




\begin{tabular}{|l|l|l|l|l|}
\hline $\begin{array}{l}\text { Wang et } \\
\text { al. } 2010\end{array}$ & $\begin{array}{l}\text { Se encontraron polifenoles en el tejido de la } \\
\text { próstata de todos los sujetos en el grupo de } \\
\text { té verde. Disminución proteína NkB-a } \\
\text { Indujeron apoptosis celular. }\end{array}$ & Sí & Próstata & $\mathrm{P}<0,05$ \\
\hline $\begin{array}{l}\text { Tsao et al. } \\
2009\end{array}$ & $\begin{array}{l}\text { Mayor respuesta clínica en los brazos de } \\
\text { la intervención combinados. Aumento de la } \\
\text { respuesta histológica dependiente de la } \\
\text { dosis. }\end{array}$ & Sí & Oral & $\mathrm{P}=0,09$ \\
\hline $\begin{array}{l}\text { McLarty et } \\
\text { al. } 2009\end{array}$ & $\begin{array}{l}\text { Reducción significativa en los niveles } \\
\text { séricos de PSA y otros marcadores } \\
\text { prostáticos. Sí }\end{array}$ & Próstata & $\mathrm{P}<0,03$ \\
\hline
\end{tabular}

\section{Discusión y conclusiones}

En la presente revisión se han identificado 14 artículos que estudian la ingesta del té y su relación con el cáncer. Los resultados sugieren una relación protectora entre la ingesta de té durante distintos períodos de tiempo y una disminución de los marcadores tumorales en distintos tipos de cánceres.

Así pues, los resultados en base al tumor estudiado indicaron que, para el cáncer de mama, la suplementación con una dosis alta de EGCG durante 12 meses en mujeres con alto riesgo de sufrir este cáncer no reduce las medidas de densidad mamográfica en todas las todas las mujeres posmenopáusicas (Samavat et al. 2017). Este resultado parece estar relacionado con la edad, ya que el estudio de Crew et al. (2015) presentó resultados similares en la reducción de la densidad mamográfica en las mujeres de 50 a 55 años. En cuanto a los cánceres ginecológicos, Garcia et al. (2014) realizaron un ensayo clínico en mujeres con infección del virus del papiloma humano para evaluar el efecto del té en la prevención de la aparición del cáncer cervical y llegaron a la conclusión, tras cuatro meses de intervención, que el té no promueve la eliminación de las lesiones precursoras del cáncer cervical.

Con respecto al cáncer de colón, Henning et al. (2013) realizaron un estudio experimental del consumo de té verde y negro en hombres, donde se observó que el té verde puede ayudar a disminuir la incidencia de problemas de colon.

Así pues, por lo que respecta al cáncer oral, Tsao et al. (2009) desarrollaron un estudio en un grupo de pacientes con lesiones premalignas orales con 3 concentraciones diferentes de té verde. La tasa de respuesta histológica fue mayor en los 3 brazos combinados; sin embargo, la tasa de respuesta clínica fue dependiente de la dosis. La intervención no mejoró la supervivencia libre de cáncer oral.

Para el cáncer de próstata, Henning et al. (2015) y Wang et al. (2010) evaluaron el consumo de 6 tazas de té verde en hombres con cáncer de próstata antes de la prostatectomía. Ambos observaron una disminución de los marcadores y una captación de polifenoles en la glándula prostática después de una intervención de té verde a corto plazo. Además, Henning et al. (2015) evaluaron también el consumo de té negro sin encontrar efectos significativos. Por otro lado, Nguyen et al. (2012), Thomas et al. (2014) y McLarty et al. (2009) analizaron el efecto de las cápsulas orales a base de té verde en pacientes con cáncer de próstata antes de la prostatectomía. Los resultados de disminución de marcadores prostáticos y de aumento de la biodisponibilidad de los polifenoles en este tejido obtenidos por Nguyen et al. (2012) no alcanzaron significación estadística. Sin embargo, el suplemento oral evaluado por Thomas et al. (2014) estaba compuesto por otros agentes fitoterapéuticos además de té verde. En este caso, igual que en el estudio realizado por McLarty et al. (2009), sí 
que observaron una disminución significativa de los marcadores prostáticos pero no evaluaron la presencia de polifenoles en el tejido prostático. Al contrario que en los anteriores, Kumar et al. (2015) realizaron un ensayo aleatorizado en hombres con dos lesiones precursoras del cáncer de próstata. No se observaron diferencias significativas en los marcadores en las dos lesiones premalignas evaluadas conjuntamente, pero sí se observó una disminución significativa de estos marcadores prostáticos al analizarlas por separado. Por tanto, el té verde puede ser beneficioso en el tratamiento del cáncer de próstata.

Por tanto, un alto consumo de té podría estar relacionado con una disminución en los marcadores tumorales y en el riesgo de recurrencia y mortalidad del cáncer de próstata en población con lesiones precursoras o diagnóstico de esta patología oncológica. El consumo de té podría ejercer también un efecto protector más moderado en otros tipos de cáncer como el colorrectal, mama y oral. En cambio, no se han encontrado resultados que encuentren una relación beneficiosa entre el té y el cáncer de cérvix y ovario. Se requieren mayores tamaños de muestra e intervenciones a más largo plazo, orientando así las futuras líneas de investigación.

\section{Referencias bibliográficas}

Cabrera, Carmen, Rafael Giménez y María Carmen López. 2003. Determination of tea components with antioxidant activity. Journal of Agricultural and Food Chemistry, 51(15): 4427-4435. https://pubs.acs.org/doi/abs/10.1021/jf0300801

Crew, Katherine D. et al. 2015. «Effects of a green tea extract, Polyphenon E, on systemic biomarkers of growth factor signalling in women with hormone receptor-negative breast cáncer». Journal of Human Nutrition and Dietetics, 28(3): 272-282. https://doi.org/10.1111/jhn.12229

Garcia, Francisco, Terri Cornelison, Tomas Nuño, David Greenspan, Jhon Byron, Chiu-Hsieh Hsu, David Alberts y Sherry Chow. 2014. "Results of a phase II randomized, double-blind, placebo-controlled trial of Polyphenon $E$ in women with persistent high-risk HPV infection and low-grade cervical intraepithelial neoplasia». Gynecologic Oncology, 132(2): 377-382. https://doi.org/10.1016/j.ygyno.2013.12.034

Henning, Susanne, Piwen Wang, Jonathan Said, Min Huang, Tristan Grogan, David Elashoff, Catherine Carpenter, David Heber y William Aronson. 2015. «Randomized clinical trial of brewed green and black tea in men with prostate cancer prior to prostatectomy». The Prostate, 75(5): 550-559. https://doi.org/10.1002/pros.22943

Henning, Susanne, Piwen Wang, Narine Abgaryan, Roberto Vicinanza, Daniela Moura de Oliveira, Yanjun Zhang, Ru-Po Lee, Catherine Carpenter, William Aronson y David Heber. 2013. "Phenolic acid concentrations in plasma and urine from men consuming green or black tea and potential chemopreventive properties for colon cancer». Molecular Nutrition \& Food Research, 57(3): 483-493. https://doi.org/10.1002/mnfr.201200646

Khan, Naghma y Hasan Mukhtar. 2018. «Tea Polyphenols in Promotion of Human Health». Nutrients, 11(1). https://doi.org/10.3390/nu11010039

Kumar, Nagi, Julio Pow-Sang, Kathleen Egan, Philippe Spiess, Shohreh Dickinson, Raoul Salup, Mohamed Helal, Jerry McLarty, Christopher Williams, Fred Schreiber y Howard Parnes. 2015. «Randomized, Placebo-Controlled Trial of Green Tea Catechins for Prostate Cancer Prevention». Cancer Prevention Research, 8(10): 879-887. https://doi.org/10.1158/1940-6207.CAPR-14-0324

Nguyen, Mike, Frederick Ahmann, Raymond Nagle, Chiu-Hsieh Hsu, Joseph Tangrea, Howard Parnes y Mitchell Sokoloff. 2012. «Randomized, double-blind, placebocontrolled trial of polyphenon $\mathrm{E}$ in prostate cancer patients before 
prostatectomy: evaluation of potential chemopreventive activities». Cancer Prevention Research, 5(2): 290-298. https://doi.org/10.1158/1940-6207.CAPR11-0306

McLarty, Jerry, Rebeca Bigelow, Mylinh Smith, Don Elmajian, Murali Ankem y James Cardelli. 2009. "Tea polyphenols decrease serum levels of prostate-specific antigen, hepatocyte growth factor, and vascular endothelial growth factor in prostate cancer patients and inhibit production of hepatocyte growth factor and vascular endothelial growth factor in vitro». Cancer Prevention Research, 2(7): 673-682. https://doi.org/10.1158/1940-6207.CAPR-08-0167

Mukhtar, Hasan y Nihal Ahmad. 2000. «Tea polyphenols: Prevention of cancer and optimizing health». American Journal of Clinical Nutrition, 71(6): 1698S-1702S.

Peterson, Julia J., Johanna Dwyer, Paul Jacques, William Rand, Ronald Prior y Kenneth Chui. 2004. "Tea variety and brewing techniques influence flavonoid content of black tea». Journal of Food Composition and Analysis, 17(3-4): 397405. https://doi.org/10.1016/j.jfca.2004.03.022

Samavat, Hamed, Giske Ursin, Tim Emory, Eunjung Lee, Renwei Wang, Carolyn Torkelson, Allison Dostal, Karen Swenson, Chap Le, Chung Yang, Mimi Yu, Douglas Yee, Anna Wu, Jian-Min Yuan y Mindy Kurzer. 2017. «A Randomized Controlled Trial of Green Tea Extract Supplementation and Mammographic Density in Postmenopausal Women at Increased Risk of Breast Cancer». Cancer Prevention Research, 10(12): 710-718. https://doi.org/10.1158/19406207.CAPR-17-0187

Thomas, Robert, Madeleine Williams, Harbinder Sharma, Aasem Chaudry y Pat $\mathrm{H}$. Bellamy. 2014. «A double-blind, placebo-controlled randomised trial evaluating the effect of a polyphenol-rich whole food supplement on PSA progression in men with prostate cancer -the UK NCRN Pomi-T study». Prostate Cancer and Prostatic Diseases, 17(2): 180-186. https://doi.org/10.1038/pcan.2014.6

Trudel, Dominique, David Labbé, Monica Araya-Farias, Alain Doyen, Laurent Bazinet, Thierry Duchesne, Marie Plante, Jean Gregoire, Marie Renaud, Berand Dimcho y Isabelle Bairati. 2013. "A two-stage, single-arm, phase II study of EGCGenriched green tea drink as a maintenance therapy in women with advanced stage ovarian cancer». Gynecologic Oncology, 131(2): 357-361. https://doi.org/10.1016/j.ygyno.2013.08.019

Tsao, Anne, Diane Liu, Jack Martin, Xi-ming Tang, Adel El-Naggar, Ignacio Wistuba, Kirk Culotta, Li Mao, Waun Hong y Vassiliki Papadimitrakopoulou. 2009. «Phase II randomized, placebo-controlled trial of green tea extract in patients with high-risk oral premalignant lesions». Cancer Prevention Research, 2(11): 931-941. https://doi.org/10.1158/1940-6207.CAPR-09-0121

Van Die, Dianne, Scott Williams, Jon Emery, Kerry Bone, Jeremy Taylor, Elizabeth Lusk y Marie Pirotta. 2017. «A Placebo-Controlled Double-Blinded Randomized Pilot Study of Combination Phytotherapy in Biochemically Recurrent Prostate Cancer». The Prostate, 77(7): 765-775. https://doi.org/10.1002/pros.23317

Wang, Piwen, William Aronson, Min Huang, Yanjun Zhang, Ru-Po Lee, David Heber y Susanne Henning. 2010. "Green tea polyphenols and metabolites in prostatectomy tissue: implications for cancer prevention». Cancer Prevention Research, 3(8): 985-993. https://doi.org/10.1158/1940-6207.CAPR-09-0210

Xue, Kathy, Lili Tang, Qingsong Cai, Ye Shen, Jianjia Su y Jia-Sheng Wang. 2015. «Mitigation of Fumonisin Biomarkers by Green Tea Polyphenols in a High-Risk Population of Hepatocellular Carcinoma». Scientific Reports, 5(1): 17545. https://doi.org/10.1038/srep17545 